\title{
Degree of handedness, but not direction, is a systematic predictor of cognitive performance
}

\author{
Eric Prichard ${ }^{1}$, Ruth E. Propper ${ }^{2}$ and Stephen D. Christman ${ }^{1}$ * \\ ${ }^{1}$ Department of Psychology, University of Toledo, Toledo, OH, USA \\ ${ }^{2}$ Department of Psychology, Montclair State University, Montclair, NJ, USA
}

\section{Edited by:}

Onur Gunturkun, Ruhr University

Bochum, Germany

Reviewed by:

Karen L. Bales, University of

California Davis, USA

Marco Steinhauser, Catholic

University of Eichstätt-Ingolstadt,

Germany

*Correspondence:

Stephen D. Christman, Department of

Psychology, University of Toledo,

Toledo, OH 43606, USA.

e-mail:stephen.christman@

utoledo.edu
A growing body of evidence is reviewed showing that degree of handedness (consistent versus inconsistent) is a more powerful and appropriate way to classify handedness than the traditional one based on direction (right versus left). Experimental studies from the domains of episodic memory retrieval, belief updating/cognitive flexibility, risk perception, and more are described. These results suggest that inconsistent handedness is associated with increased interhemispheric interaction and increased access to processes localized to the right cerebral hemisphere.

Keywords: handedness, interhemispheric interaction, episodic memory, belief updating, cognitive flexibility

\section{INTRODUCTION}

Psychological research examining "individual differences" grounded in biology (in contrast with, for example, personality/temperament, or in experience) typically focuses on sex and age. Another biologically based dimension of individual differences, handedness, has received much less attention. This neglect has arisen in part because handedness research has largely been the province of neuropsychologists, and such research makes little contact with the methods and theories of mainstream psychology. This lack of contact is the product of both the idiosyncratic methods employed in handedness research (e.g., lateralized presentation of input), and the fact that, historically, research attempting to identify key functional and structural differences between leftand right-handers has produced equivocal results (other than the fact that brain asymmetry appears to be weaker and more variable in left-handers).

The purpose of this article is to acquaint the reader with a growing body of evidence identifying handedness as a robust predictor of individual differences across a number of domains. The research to be reviewed breaks with past handedness research in a critical way: instead of comparing left- versus right-handedness, it focuses on comparisons between consistent/strong-handers $(\mathrm{CH})$ and inconsistent/mixed-handers (ICH). Here, we define $\mathrm{CH}$ as using the dominant hand for virtually all common manual activities, and ICH as using the non-dominant hand for at least one common manual activity. That is, historically, research examining individual differences in handedness focused on the effects of direction of hand preference on behavior, thereby comparing left versus right-handers. However, evidence has accumulated that the critical dimension on which the handedness groups differ is in degree (consistent versus inconsistent) of hand preference. That is, how consistently, or strongly, an individual prefers to use one versus the other hand over a wide variety of tasks may be the more appropriate indicator of cerebral organization and of behavior. In fact, we would argue here that a major reason why previous research has failed to clearly determine individual differences in handedness effects on behavior is because the measure used to define handedness has heretofore been incorrect. Instead of direction of hand preference being the variable of interest, it should be degree.

The distinction between consistent and inconsistent handedness is based on a simple median split on scores on the Edinburgh Handedness Inventory (Oldfield, 1971). Scores range from -100 (pure left handed) to +100 (pure right-handed). The population median, based on a large sample of 1595 subjects, is 80. Thus, inconsistent handedness is defined as handedness scores below 80 , which is equivalent to performing at least one of the ten activities with the non-dominant hand. A summary of the population proportions of handedness is presented in Table 1.

There are two things to note about Table 1. First, right-handers tend to be consistent handed while left-handers are largely inconsistent handed. Second, consistent handedness is more prevalent among females than among males.

While it is beyond the scope of this chapter to fully explicate the mechanisms underlying the distinction between $\mathrm{CH}$ and $\mathrm{ICH}$, key principles involve interhemispheric communication and functional access to right hemisphere processing. More consistent hand preference is associated with smaller corpus callosum size (e.g., Luders et al., 2010) and with decreased right hemisphere activation (e.g., Propper et al., 2012). Accordingly, consistentversus inconsistent handedness is associated with decreased versus increased interhemispheric interaction and with decreased versus increased right hemisphere access, respectively. The following review will focus primarily on two task domains for which interhemispheric interaction and right hemisphere access have 
Table 1 | Percentages of female and male participants, classified according to both direction and degree of handedness.

\begin{tabular}{lrrr}
\hline Direction of handedness & \multicolumn{3}{c}{ Degree of handedness } \\
\cline { 2 - 4 } & Strong & Mixed \\
\hline FEMALES & 59.3 & 31.0 & 90.3 \\
Right & 3.2 & 6.5 & 9.7 \\
Left & 62.5 & 37.5 & \\
MALES & & & \\
Right & 47.8 & 41.6 & 89.4 \\
Left & 2.4 & 8.2 & 10.6 \\
& 50.2 & 49.8 & \\
\hline
\end{tabular}

been implicated: episodic memory retrieval (associated with right frontal areas) and belief updating/cognitive flexibility (associated with right frontal-parietal areas), with the evidence showing that ICH exhibit superior episodic memory and increased belief updating/cognitive flexibility. Other related findings will also be presented. A summary of the findings reviewed is provided in Table 2.

We are not arguing that the reader should become a "handedness" researcher. Instead, we are encouraging researchers to include degree of handedness as a variable in their designs, much like many already do with sex and/or age. At the very least, including handedness as a variable in analyses would move variability out of the omnibus error term and into a specific effect term, thereby providing increased statistical power to detect other effects of primary interest. At best, systematic individual differences as a function of handedness and concomitant gradations in interhemispheric interaction and in right hemisphere access, could prove to be a useful construct in the development and testing of domain-specific theories.

\section{HANDEDNESS AND MEMORY}

Some of the most robust findings demonstrating the effects of handedness as an individual difference variable come from the domain of memory research. This work initially relied on predictions made by the Hemispheric Encoding and Retrieval and Asymmetry (HERA) model (Tulving et al., 1994). They reported that, for semantic memory tasks, brain activity at both encoding and retrieval were lateralized to the left hemisphere. In contrast, for episodic memory, activation at encoding versus retrieval was lateralized to the left versus right hemispheres, respectively. This finding raised the possibility that (i) episodic memory relies on interhemispheric interaction (necessary to integrate left hemisphere encoding with right hemisphere retrieval) to a greater extent than does semantic memory (left hemisphere encoding and retrieval); (ii) individual differences in interhemispheric interaction would be reflected in individual differences in memory ability, and (iii) individual differences in degree of hand preference, being associated with individual differences in interhemispheric interaction, would therefore also be associated with individual differences in memory performance. Specifically, inconsistently handed individuals, having increased interhemispheric interaction, possibly mediated via
Table 2 | Summary of research on handedness differences in memory.

\begin{tabular}{|c|c|c|}
\hline Task & Findings & Citation \\
\hline Free recall of words & $\mathrm{ICH}$ advantage & Propper et al. (2005) \\
\hline Free recall of words & $\mathrm{ICH}$ advantage & Lyle et al. (2008a) \\
\hline Free recall of words & $\mathrm{ICH}$ advantage & $\begin{array}{l}\text { Christman and Butler } \\
\text { (2011) }\end{array}$ \\
\hline $\begin{array}{l}\text { Free recall of events from } \\
\text { own life }\end{array}$ & $\mathrm{ICH}$ advantage & Propper et al. (2005) \\
\hline $\begin{array}{l}\text { Recall of early childhood } \\
\text { memories }\end{array}$ & $\mathrm{ICH}$ advantage & Christman et al. (2006b) \\
\hline Paired-associate recall & $\mathrm{ICH}$ advantage & Lyle et al. (2008b) \\
\hline Source memory (DRM & $\mathrm{ICH}$ advantage & Christman et al. (2004) \\
\hline
\end{tabular}

paradigm)

Source memory (sensory

$\mathrm{ICH}$ advantage

Lyle et al. (2008b)

modality)

Self-reported everyday

memory

Self-reported dream recall

Incidental memory for

deeply processed words

Incidental memory for

shallowly processed words

Know versus remember

judgments

Word recognition

Word recognition

Face memory

Implicit memory

Semantic memory

Memory for paragraphs

$\mathrm{ICH}$ advantage

$\mathrm{ICH}$ advantage

ICH advantage

No difference

Christman and Propper (2008)

Christman (2007)

Christman and Butler

(2011)

Christman and Butler

(2011)

$\mathrm{ICH}:$ rem $>$ know

$\mathrm{CH}$ : rem $=$ know

Propper and Christman (2004)

No difference

Propper and Christman (2004)

No difference Lyle et al. (2008a)

$\mathrm{ICH}$ advantage

No difference

No difference

Lyle and Orsborn (2011)

Propper et al. (2005)

Propper et al. (2005)

$\mathrm{ICH}$ advantage

Prichard and Christman

Openness to persuasion

Gullibility

Belief in evolution

Magical ideation

Cognitive dissonance

$\mathrm{ICH}$ more open

(2012)

Christman et al. (2008)

$\mathrm{ICH}$ more gullible

Christman et al. (2008)

$\mathrm{ICH}$ more likely

$\mathrm{ICH}$ have higher

levels

Niebauer et al. (2004)

Barnett and Corballis

(2002)

ICH have higher Jasper et al. (2009)

levels

Placebo effect

Anchoring effect

Larger in $\mathrm{ICH}$

Christman et al. (2006a)

Larger in $\mathrm{ICH}$

Jasper and Christman

(2005)

Counterfactuals

$\mathrm{ICH}$ produce

Jasper et al. (2008)

more

Ambiguous figures

$\mathrm{ICH}$ higher

Christman et al. (2009)

reversal rate

Ambiguous words

Greater activation

in $\mathrm{ICH}$

Sontam and Christman

Musical preferences

Greater

(2012)

preference for

obscure genres in

$\mathrm{ICH}$

Sensation seeking

Higher levels in

Christman (2011a)

$\mathrm{ICH}$ 


\section{Table 2 | Continued}

\begin{tabular}{lll}
\hline Task & Findings & Citation \\
\hline Consumer loyalty & Lower levels in & Lanning and Christman \\
& ICH & (2010) \\
Right Wing & Lower levels in & Christman (2008) \\
Authoritarianism & ICH & \\
Sense of disgust & Stronger in CH & Christman (2012) \\
Risk perception & ICH more loss & Christman et al. (2007b) \\
& averse & \\
Sunk cost effect & Higher levels in & Westfall et al. (2012) \\
& ICH & \\
Taking others' perspectives & ICH are better & Sontam et al. (2005); \\
& & Lanning and Christman \\
& & (2010) \\
Sleep architecture & ICH: shorter & Propper et al. (2007) \\
& latency, increased & \\
& time in NREM & \\
\hline
\end{tabular}

$\mathrm{ICH}$, inconsistent handed; $\mathrm{CH}$, consistent handed.

greater corpus callosal connectivity, would demonstrate superior episodic, but not semantic, memory.

Supporting the hypothesis, Propper et al. (2005) found that $\mathrm{ICH}$ outperformed $\mathrm{CH}$ on an episodic memory task involving word list recall. Interestingly, there was no significant difference between the handedness groups on a word fragment completion task used as a test of semantic memory. Handedness differences are not typically found in recognition memory; however, in another test of episodic memory (e.g., Christman and Propper, 2001), Propper and Christman (2004) found that, despite equal levels of recognition memory, $\mathrm{ICH}$ are more likely to report explicitly episodically "remembering" an item while $\mathrm{CH}$ are more likely to report merely semantically "knowing" that they saw the item before.

The findings that ICH have superior episodic recall abilities and show a greater tendency than $\mathrm{CH}$ to make "remember" judgments raises the possibility that handedness differences in episodic memory may reflect underlying differences in source memory. Three findings examining handedness differences in false alarms, using both laboratory based and real-world memories, support this notion. First, Christman et al. (2004) demonstrated that ICH are less likely to report having a false memory for the critical lure in by the Roediger and McDermott (1995) paradigm, suggesting that the source of memories may be more available in the $\mathrm{ICH}$ relative the $\mathrm{CH}$. Second, Lyle et al. (2008b) tested source memory for words that participants had originally either read or heard; again, relative to $\mathrm{CH}$, ICH were better at remembering whether the original presentation of items has been visual or auditory. Finally, Lyle et al. (2008a) also reported fewer false alarms in $\mathrm{ICH}$.

These "snapshot" handedness effects on memory-that is, of superior episodic memory among $\mathrm{ICH}$ relative to $\mathrm{CH}-$ have been extended in investigations of handedness and memory effects across the lifespan. Christman et al. (2006b) reported that $\mathrm{ICH}$ experience an earlier offset of childhood amnesia, and therefore a younger age for their earliest childhood memory. Lyle et al. (2008b) obtained an ICH advantage on a recall task in a sample of middle aged adults, but not with a sample of older adults. Lyle et al. (2008b) proposed that as people age, the corpus callosum degenerates, thus attenuating the ICH advantage. Specifically, the decline in memory from middle- to older-aged adults was larger in the $\mathrm{ICH}$, consistent with a callosal contribution to episodic memory. Finally, Kempe et al. (2009) found that ICH were more easily able to acquire foreign vocabulary words in adulthood. Although vocabulary recall in adulthood may involve both episodic and semantic memory processes, this finding suggests that individual differences in handedness may account for some between individual variability in language acquisition.

Findings of superior episodic memory in $\mathrm{ICH}$ relative the $\mathrm{CH}$ extend beyond artificial, laboratory information. For example, Propper et al. (2005) demonstrated an ICH advantage for autobiographical, self-reported events that occurred outside the laboratory, and Christman et al. (2006b) reported an ICH advantage for earliest childhood memories. Christman and Propper (2008) found that ICH reported fewer memory problems in everyday life, especially in the domains of task monitoring and conversation. Christman (2007) reported that ICH remember more dreams and report more frequent déjà vu experiences. Prichard and Christman (2012) found that the ICH advantage in memory extends to recall of paragraph-level material, although the ICH advantage was larger for males than for females. Finally, Lyle and Orsborn (2011) reported superior face memory in ICH.

It is important to point out that in the studies reviewed above, most compared ICH with consistent right-handers. Given that consistent-left-handers are only about $1-3 \%$ of the population (Lansky et al., 1988), studies comparing ICH with consistently right and consistently left handed individuals are time consuming, difficult to conduct, and therefore infrequent. However, Lyle et al. (2012) collected a large sample of consistent-left-handers in order to determine whether it is consistent handedness per se that is associated with less or episodic memory, or if this effect is specific to consistent-right-handedness. Importantly, ICH outperformed $\mathrm{CH}$, on an episodic memory task, regardless of the direction of $\mathrm{CH}$ hand preference; that is, regardless of whether $\mathrm{CH}$ were left- or right-handed, ICH performed better.

\section{HANDEDNESS AND BELIEF UPDATING/COGNITIVE FLEXIBILITY}

Ramachandran (1995) hypothesized that the left hemisphere is important for maintaining our current beliefs about the world, while the right hemisphere acts as an anomaly detector and is sensitive to information inconsistent with those beliefs. This suggests a possible role for interhemispheric connectivity in the belief updating process. When something challenges pre-existing beliefs, it may be the right hemisphere's job to notice the inconsistency and communicate it to the left hemisphere. Since belief updating may be considered, more broadly, an example of cognitive flexibility, further studies have also looked at numerous DVs which, taken together, may be considered measures of cognitive flexibility. It is to the literature investigating a possible relationship between handedness and belief updating/cognitive flexibility which we now turn. 
Niebauer et al. (2004) found that consistent-handers are more likely to report holding young-earth creationist beliefs. The authors argued that, because children typically hold creationist views at some point (Evans, 2000), the retention of such beliefs is the result of a failure to update beliefs about human origins in light of new evidence. Similarly, Christman et al. (2008) reported that ICH are more open to persuasion. At the same time, however, they found that ICH were also more gullible, showing greater susceptibility to the "Barnum effect." This finding may be related to that of Barnett and Corballis (2002), who reported that ICH were more prone to magical ideation (i.e., beliefs in ESP, UFOs, astrology, etc). Thus, $\mathrm{CH}$ are more resistant to belief updating, and are therefore less likely to alter their views based on little evidence.

Once researchers obtained the basic finding that degree of handedness predicts the tendency to update one's beliefs or, to frame it differently, degree of handedness predicts resistance to information challenging pre-existing beliefs, the handedness paradigm has been applied to several areas for which the belief updating/cognitive flexibility process is relevant. For example, it has been applied to cognitive dissonance (Jasper et al., 2009), who conducted a study in which participants were given false personality profiles. In the experimental condition, participants were told their profiles indicated high levels of sexism. When asked to judge a fictional sex based discrimination suit, $\mathrm{ICH}$ who had been told they were sexist awarded higher payouts than $\mathrm{CH}$, indicating greater cognitive dissonance in ICH. Handedness differences have also been obtained in the magnitude of placebo effects, with $\mathrm{ICH}$ exhibiting much larger placebo effects than $\mathrm{CH}$ (Christman et al., 2006a). Thus, handedness may be a variable of interest for researchers examining how belief affects treatment outcomes, or for researchers who want to reduce the error term in clinical trials that necessarily include a placebo condition.

As stated at the beginning of the section, belief updating could arguably fall under the broader area of cognitive flexibility. The empirical evidence indicates handedness does indeed predict cognitive flexibility as measured by a surprising variety of DVs. Starting with research on basic heuristics, Jasper and Christman (2005) found that inconsistent-handers were less susceptible to anchoring on a task that asked participants 12 factual knowledge questions after exposing them to unhelpful high or low anchors. Resisting anchors in such a situation may require one to hold multiple representations, a process requiring considerable cognitive flexibility. In the area of counterfactual reasoning, Jasper et al. (2008) found that, when asked to come up with counterfactual alternatives to various scenarios, ICH produce more upward counterfactuals and downward counterfactuals. Research on more basic perceptual processes shows that ICH can more easily update their perception of ambiguous figures (Christman et al., 2009) and that ICH more readily fall for a sensory illusion in which a participant comes to "feel" taps on a fake arm (Niebauer et al., 2002). During investigations of semantic flexibility, ICH showed a greater tendency to switch between subcategories when asked to name as many animals as they could (Sontam et al., 2009) and had an easier time accessing "weak" associates of ambiguous stimulus words than consistent-handers did (Sontam and Christman, 2012). ICH have also been shown to be more creative, measured via divergent thinking, compared to $\mathrm{CH}$ (Shobe et al., 2009).
As with the handedness and memory paradigms, there has been an interest in whether these cognitive flexibility effects generalize beyond the realm of interesting experiments. What does it mean to say inconsistent-handers are more cognitively flexible outside of an experimental context? As it turns out, handedness predicts certain kinds of esthetic judgments, with ICH showing more appreciation for self-referential works by M.C. Escher (Niebauer and Garvey, 2004) and for a wider variety of musical genres (Christman, 2013) than CH. Further, consistent-handers are less sensation seeking (Christman, 2011a), exhibit greater consumer brand loyalty (Christman and Lanning, 2012), have greater disgust sensitivity (Christman, 2012), and score higher on measures of Right Wing Authoritarianism (Christman, 2008) than ICH.

Perhaps of the greatest practical relevance, the link between handedness and cognitive flexibility is of potential clinical relevance. Consistent-handers are more likely than inconsistenthanders to ruminate (Niebauer, 2004), to display eating disorder symptomatology (Christman et al., 2007a), and to show higher levels of body dysmorphia (Christman, 2011b).

\section{MISCELLANEOUS HANDEDNESS FINDINGS}

While the memory and cognitive flexibility literatures are the most well developed of the literatures investigating degree of handedness as an individual difference variable, it is worth mentioning several empirical studies that have branched out beyond these two major areas. Although much remains to be explained about what underlies the following findings, it is hoped that there will be something of interest to researchers from across the discipline of psychology.

Several studies looking at emotion and risk perception have uncovered evidence of handedness effects. Propper et al. (2010) reported that $\mathrm{ICH}$ demonstrated increased negative affect across a wide variety of emotional states, compared to $\mathrm{CH}$, although only feelings of "anger" were significantly greater in ICH. Christman et al. (2007b) found that, when making risky decisions, inconsistent-handers reported being more influenced by the perceived risks of a behavior and consistent-handers reported being more influenced by the perceived benefits. Westfall et al. (2012) found that inconsistent-handers showed more inaction inertia and a greater sunk cost effect unless it was made clear that staying on a particular course would definitely result in a greater loss than abandoning it. Once it was clear that inaction would definitely result in a greater loss, there was a reversal with inconsistent-handers showing less inaction inertia. Finally, Bhattacharya et al. (2012) found that selectively activating the right hemisphere via Schiffer goggles increased the tendency for inconsistent-handers to focus on risks and consistent-handers to focus on benefits. It may be that these findings are related to a potential right hemisphere role in negative affect/withdrawal motivational states.

Handedness has also been used as a variable in traditional self-other/person perception paradigms. ICH seem to have an easier time taking other perspectives into account (Sontam et al., 2005; Lanning and Christman, 2010) and have better memory for counter-stereotypical information (Christman and Sterling, 2009). Additionally, sex and race effects on the Implicit Association Test (IAT) are modulated by handedness (Christman and 
Sahu, 2012). For example, the weakest stereotyping was exhibited by $\mathrm{ICH}$ European Americans and by $\mathrm{CH}$ African-American males.

We will wrap up the present review with several additional findings that are not yet part of any broad research program, but which may prove to be promising leads in the future. While investigating possible associations between handedness and sleep architecture, Propper et al. (2004) found that ICH had shorter sleep latency and spent more time in NREM, although Propper et al. (2007) also obtained evidence that consistent-left-handers spend more time in NREM and less time in REM than consistent-right-handers, thus raising the possibility that both strength and direction of handedness should be considered when researching sleep. Christman

\section{REFERENCES}

Barnett, K. J., and Corballis, M. C. (2002). Ambidexterity and magical ideation. Laterality 7, 75-84.

Bhattacharya, C., Jasper, J. D., Corser, R., and Christman, S. D. (2012). Selective activation of the right hemisphere changes the way we perceive risks and benefits. Presented at the 24th Annual Convention of the Association for Psychological Science, Chicago.

Christman, S., and Propper, R. (2008). Individual differences in performance on the everyday memory questionairre: Effects of degree of handedness. Presented at the 37th Annual Meeting of the International Neuropsychological Society, Waikoloa, Hawaii.

Christman, S. D. (1993). Handedness in musicians: bimanual constraints on performance. Brain Cogn. 22, 266-272.

Christman, S. D. (2007). Individual differences in déjà vu and jamais vu experiences: degree of handedness and access to the right hemisphere. Presented at the 19th Annual Meeting of the Association for Psychological Science, Washington, DC.

Christman, S. D. (2008). Individual differences in Right-Wing Authoritarianism: handedness and cognitive rigidity. Presented at the Annual Meeting of the Midwestern Psychological Association, Chicago.

Christman, S. D. (2011a). Handedness and cognitive rigidity: strong righthanders report less sensation seeking. Presented at the Annual Meeting of the Midwestern Psychological Association, Chicago.

Christman, S. D. (2011b). Individual differences in body dysmorphic disorder: Handedness is more important than sex. Presented at the 40th Annual Meeting of the International Neuropsychological Society, Boston.

Christman, S. D. (2012). Strong degrees of right-handedness are associated with stronger sense of disgust. Presented at the Annual Meeting of the Midwestern Psychological Association, Chicago.

Christman, S. D. (2013). Handedness and 'earedness': strong righthanders are less likely to prefer obscure musical genres. Psychol. Music 41, 89-96.

Christman, S. D., Bentle, M., and Niebauer, C. L. (2007a). Handedness differences in body image distortion and eating disorder symptomatology. Int. J. Eat. Disord. 40, 247-256.

Christman, S. D., Jasper, J. D., Sontam, V., and Cooil, B. (2007b). Individual differences in risk perception versus risk taking: Handedness and interhemispheric interaction. Brain Cogn. 63, 51-58.

Christman, S. D., and Butler, M. (2011). Mixed-handedness advantages in episodic memory obtained under conditions of intentional learning Cogn. 77, 17-22.

Christman, S. D., Geers, A. L., Kosbab, K., and Weiland, P. E. (2006a). Placebos and belief updating: Strong right-handers do not show placebo effects. Presented at the 35th Annual Meeting of the International Neuropsychological Society, Boston.

Christman, S. D., Propper, R. E., and Brown, T. J. (2006b). Increased interhemispheric interaction is associated with earlier offset of childhood amnesia. Neuropsychology 20, 336-345.

Christman, S. D., Henning, B., Geers, A. L., Propper, R. E., and Niebauer, C. L. (2008). Mixed-handed persons are more easily persuaded and are more gullible: Interhemispheric interaction and belief updating. Laterality 13, 403-426.

Christman, S. D., and Lanning, M. D. (2012). Individual differences in edness and cognitive flexibility. extend to incidental learning. Brain consumer brand favoritism: Hand-

(1993) discovered a compelling example of how degree of handedness may be related to preferences for certain motor tasks when he surveyed musicians and found that ICH were more likely to play instruments that require temporally integrated bimanual motor actions.

In conclusion, the studies reviewed above demonstrate a robust and systematic effect of degree of handedness in two well defined domains; episodic memory retrieval and belief updating/cognitive flexibility, and in other areas as well, including emotion and sleep architecture. It is hoped that this review will inspire a wider body of psychology investigators to incorporate this long neglected and misunderstood dimension of human individual difference into their research.

Presented at the 24th Annual Convention of the Association for Psychological Science, Chicago.

Christman, S. D., and Propper, R. E. (2001). Superior episodic memory is associated with interhemispheric processing. Neuropsychology 15, 607-616.

Christman, S. D., Propper, R. E., and Dion, A. (2004). Increased interhemispheric interaction is associated with decreased false memories in a verbal converging semantic associates paradigm. Brain $\operatorname{Cogn}$. 56, 313-319.

Christman, S. D., Sontam, V., and Jasper, J. D. (2009). Individual differences in ambiguous figure perception: degree of handedness and interhemispheric interaction. Perception 38, 1183-1198.

Christman, S. D., and Sterling, M. (2009). Individual differences in person perception: mixed-handers have better memory for counterstereotypical information. Presented at the 50th Annual Meeting of the Psychonomic Society, Boston.

Christman, S.D., and Sahu, A. (2012). Individual differences in the IAT: Handedness modulates effects of sex and race. Presented at the 24th Annual Convention of the Association for Psychological Science, Chicago.

Evans, E. M. (2000). The emergence of beliefs about the origins of species in school-age children. Merrill Palmer Q. 46, 19-52.

Jasper, J. D., Barry, K., and Christman, S. D. (2008). Individual differences in counterfactual production. Pers. Individ. Dif. 45, 488-492.

Jasper, J. D., and Christman, S. D. (2005). A neuropsychological dimension for anchoring effects. J. Behav. Decis. Mak. 18, 343-369.

Jasper, J. D., Prothero, M., and Christman, S. D. (2009). I'm not sexist!!! Cognitive dissonance and the differing cries of mixed- and strong-handers. Pers. Individ. Dif. 47, 268-272.

Kempe, V., Brooks, P. J., and Christman, S. D. (2009). Inconsistent handedness is linked to more successful foreign language vocabulary learning. Psychon. Bull. Rev. 16, 480-485.

Lanning, M. D., and Christman, S. D. (2010). Handedness and 'above/below' average effects: Strong right-handers are poorer at taking others' perspectives into account. Presented at the 51st Annual Meeting of the Psychonomic Society, St. Louis. Lansky, L. M., Feinstein, H., and Peterson, J. M. (1988). Demography of handedness in two samples of randomly selected adults $(N=2083)$. Neuropsychologia 26, 465-477.

Luders, E., Cherbuin, N., Thompson, P. M., Gutman, B., Anstey, K. J., Sachdev, P., et al. (2010). When more is less: associations between corpus callosum size and handedness lateralization. Neuroimage 52, 43-49.

Lyle, K. B., Hanaver-Torrez, S. D., Hacklander, R. P., and Edlin, J. M. (2012). Consistency of handedness, regardless of direction, predicts baseline memory accuracy and potential for memory enhancement. $J$. Exp. Psychol. Learn. Mem. Cogn. 38, 187-193.

Lyle, K. B., Logan, J., and Roediger, H. L. (2008a). Eye movements enhance memory for individuals who are strongly right-handed and harm it for individuals who are not. Psychon. Bull. Rev. 15, 515-520.

Lyle, K. B., McCabe, D. P., and Roediger, H. L. (2008b). Handedness is related to memory via hemispheric interaction: evidence from paired associated recall and source memory tasks. Neuropsychology 22, 523-530.

Lyle, K. B., and Orsborn, A. E. (2011). Inconsistent handedness and saccade execution benefit face memory without affecting interhemispheric interaction. Memory 19, 613-624. 
Niebauer, C. L. (2004). Handedness and the fringe of consciousness: Strong handers ruminate while mixed handers self-reflect. Conscious. Cogn. 13, 730-745.

Niebauer, C. L., Aselage, J., and Schutte, C. (2002). Interhemispheric interaction and consciousness: degree of handedness predicts the intensity of a sensory illusion. Laterality 7, 85-96.

Niebauer, C. L., Christman, S. D., Reid, S. A., and Garvey, K. (2004). Interhemispheric interaction and beliefs on our origin: degree of handedness predicts beliefs in creationism versus evolution. Laterality 9, 433-447.

Niebauer, C. L., and Garvey, K. (2004). Gödel, Escher and degree of handedness: differences in interhemispheric interaction predict differences in understanding self-reference. Laterality 9, 91-113.

Oldfield, C. (1971). The assessment and analysis of handedness: the Edinburgh inventory. Neuropsychologia 9, 97-113.

Prichard, E., and Christman, S.D. (2012). Individual differences in episodic memory: sex differences are confined to strongly-handed individuals. Presented at the 24th Annual Convention of the Association for Psychological Science, Chicago.

Propper, R. E., Brunyé, T. T., Christman, S. D., and Bologna, J. (2010). Negative emotional valence is associated with non-right-handedness and increased imbalance of hemispheric activation as measured by tympanic membrane temperature. J. Nerv. Ment. Dis. 198, 691-694.

Propper, R. E., and Christman, S. D. (2004). Mixed- versus stronghandedness is associated with biases toward 'Remember' versus 'Know' judgments in recognition memory: role of interhemispheric interaction. Memory 12, 707-714.

Propper, R. E., Christman, S. D., and Olejarz, S. (2007). Home-recorded sleep architecture as a function of handedness II: consistent right- versus consistent left-handers. J. Nerv. Ment. Dis. 95, 689-692.

Propper, R. E., Christman, S. D., and Phaneuf, K. A. (2005). A mixedhanded advantage in episodic memory: A possible role of interhemispheric interaction. Mem. Cognit.33, 751-757.

Propper, R. E., Lawton, N., Przyborski, M., and Christman, S. (2004). A naturalistic assessment of sleep architecture as a function of handedness using a home sleep monitor. Brain Cogn. 54, 186-197.

Propper, R. E., Pierce, J., Geisler, M. W., Christman, S. D., and Bellorado, N. (2012). Hemispheric asymmetry in frontal EEG: Inconsistentright-handers are more right hemisphere active. Open J. Med. Psychol. 1, 86-90.
Ramachandran, V. S. (1995). Anosognosia in parietal lobe syndrome. Conscious. Cogn. 4, 22-51.

Roediger, H. L., and McDermott, K. B. (1995). Creating false memories: Remembering words not presented on a list. J. Exp. Psychol. Learn. Mem. Cogn. 21, 803-814.

Shobe, E. R., Ross, N. M., and Fleck, J. I. (2009). Influence of handedness and bilateral eye movements on creativity. Brain Cogn. 71, 204-214.

Sontam, V., and Christman, S. D. (2012). Semantic organization and handedness: Mixed-handedness is associated with more diffuse activation of ambiguous word associates. Laterality $17,38-50$.

Sontam, V., Christman, S. D., and Jasper J. D. (2005). Individual differences in me v. thee effects. Presented at the 2005 Annual Meeting of the Society for Judgment and Decision Making, Toronto.

Sontam, V., Christman, S. D., and Jasper, J. D. (2009). Individual differences in semantic switching flexibility: effects of handedness. J. Int. Neuropsychol. Soc. 15, 1023-1027.

Tulving, E., Kapur, S., Craik, F. I. M., Moscovitch, M., and Houle, S. (1994). Hemispheric encoding/retrieval asymmetry in episodic memory: positron emission tomography findings. Proc. Natl. Acad. Sci. U.S.A. 91, 2016-2020.
Westfall, J., Jasper, J. D., and Christman, S. D. (2012). Inaction inertia, the sunk cost effect, and handedness: avoiding the losses of past decisions. Brain Cogn. 80, 192-200.

Conflict of Interest Statement: The authors declare that the research was conducted in the absence of any commercial or financial relationships that could be construed as a potential conflict of interest.

Received: 23 October 2012; paperpending published: 19 November 2012; accepted: 06 January 2013; published online: 31 January 2013.

Citation: Prichard E, Propper RE and Christman SD (2013) Degree of handedness, but not direction, is a systematic predictor of cognitive performance. Front. Psychology 4:9. doi: 10.3389/fpsyg.2013.00009

This article was submitted to Frontiers in Cognition, a specialty of Frontiers in Psychology.

Copyright () 2013 Prichard, Propper and Christman. This is an open-access article distributed under the terms of the Creative Commons Attribution License, which permits use, distribution and reproduction in other forums, provided the original authors and source are credited and subject to any copyright notices concerning any third-party graphics etc. 